\title{
ROBOT TELECONTROLADO APLICADO EN LA MITIGACIÓN DE INCENDIOS PARA LA ESTACIÓN DE BOMBEROS VOLUNTARIOS VICTORIA N 08, LIMA-PERÚ
}

\author{
TELECONTROLLED ROBOT USED FOR FIRE MITIGATION \\ IN VICTORIA FIRE STATION No. 08, LIMA-PERU
}

\author{
Arturo A. Morzan Soto ${ }^{1}$, Bruno G. Zerga Tapia ${ }^{2}$, \\ Ricardo J. Palomares Orihuela ${ }^{33}$
}

RECEPCIÓN: 27 DE SETIEMBRE DE 2020

ACEPTACIÓN: 29 DE OCTUBRE DE 2020

\section{RESUMEN}

La presente investigación corresponde a la aplicación de un robot telecontrolado en la mitigación de incendios para la Estación de Bomberos Voluntarios Victoria $\mathrm{N}^{\circ}$ 08 , el cual detecta zonas de alta temperatura utilizando imágenes térmicas.

Palabras clave: Telecontrolado, cámara térmica, robot

\section{ABSTRACT}

This research corresponds to the application of a remotecontrolled robot in fire mitigation for the La Victoria No. 08 Fire Station, which detects high temperature areas using thermal imaging.

Keywords: Remote control, thermal camera, robot

1 Ingeniero Mecatrónico de la Universidad Ricardo Palma, Certificado en Instalaciones Eléctricas Industriales por TECSUP, Ingeniero de Proyectos bajo el cargo de On Site Manager en Siemens Healthcare.

2 Ingeniero Mecatrónico de la Universidad Ricardo Palma, Certificado en Seguridad Industrial por BUREAU VERITAS, Certificado en Equipos de Gases Arteriales por SIEMENS HEALTH-CARE, Ingeniero de servicio en instalación de equipos de alta precisión y grado médico en BAIRES S.A.C.

3 Ingeniero Mecatrónico de la Universidad Nacional de Ingeniería, Doctor en Ciencias de la Educación. Magíster en Ciencias de la Educación con mención en Docencia Universitaria, Maestría en Ciencias de la Electrónica con mención en Ingeniería Biomédica. Miembro profesional RAS y EMBS de la IEEE. Docente de las asignaturas de Robótica, Inteligencia Artificial, Mecatrónica Medica y Diseńo Mecatrónico de la Escuela Profesional de Ingeniería Mecatrónica de la Universidad Ricardo Palma. Docente de la Carrera Profesional de Ingeniería Electrónica y Telecomunicaciones de la Universidad Nacional Tecnológica de Lima Sur. 


\section{INTRODUCCIÓN}

El Cuerpo General de Bomberos Voluntarios del Perú-CGBVP desarrolla acciones que permiten controlar incendios. Cuenta con 175 compañías en todo el país. En la figura 1, se observa que, durante 2018, ocurrieron 13729 incendios.

\begin{tabular}{|c|c|c|c|c|c|c|c|c|c|c|c|c|c|}
\hline \multirow[b]{2}{*}{ TIPO DE EMERGENCIA } & \multicolumn{10}{|c|}{$\begin{array}{l}\text { ESTADISTICA DE EMERGENCIAS ATENDIDAS A NIVEL } \\
\text { TIPO DE EMERGENCIA - } 2018\end{array}$} & \multicolumn{3}{|c|}{ NACIONAL } \\
\hline & ENE & FEB & MAR & ABR & MAY & JUN & JUL & AGO & SEP & OCT & Nov & DIC & TOTAL \\
\hline Incendios & 1272 & 1017 & 1070 & 946 & 882 & 845 & 1060 & 1208 & 1493 & 1282 & 1143 & 1511 & 13729 \\
\hline Fuga de gas licuado & 547 & 476 & 543 & 549 & 460 & 479 & 481 & 517 & 501 & 570 & 540 & 584 & 6255 \\
\hline Emergencias medicas & 5004 & 4681 & 5.446 & 5.150 & 5534 & 5264 & 6121 & 6004 & 5.527 & 5438 & 4577 & 5527 & 64273 \\
\hline Rescates & 395 & 351 & 429 & 358 & 326 & 435 & 399 & 392 & 374 & 354 & 321 & 411 & 4545 \\
\hline Derrame de productas & 3 & 7 & 5 & 9 & 6 & 6 & 12 & 21 & 14 & 8 & 8 & 21 & 120 \\
\hline Corto circuito & 248 & 213 & 241 & 241 & 243 & 225 & 244 & 233 & 233 & 209 & 196 & 256 & 2782 \\
\hline Servicios especiales & 543 & 460 & 556 & 716 & 735 & 698 & 910 & 658 & 749 & 780 & 754 & 810 & 8375 \\
\hline Accidentes vehiculares & 1267 & 1189 & 1347 & 1312 & 1306 & 1307 & 1377 & 1535 & 1435 & 1348 & 1280 & 1517 & 16220 \\
\hline Falsa alarma & 287 & 282 & 284 & 276 & 255 & 233 & 244 & 280 & 291 & 277 & 238 & 263 & 3210 \\
\hline Otros & 232 & 225 & 241 & 171 & 143 & 325 & 190 & 177 & 167 & 204 & 220 & 194 & 2489 \\
\hline TOTAL & 9798 & 8907 & 10162 & 9728 & 9898 & 9817 & 11038 & 11025 & 10784 & 10470 & 9277 & 11094 & 121998 \\
\hline
\end{tabular}

Estadisticas procesada el $1 / 1 / 2019$ a las $0: 1$ al $100 \%$
FUENTE: CUERPO GENERAL DE BOMBEROS VOLUNTARIOS DEL PERÚ

Fig. 1. Cantidad de emergencias atendidas a nivel nacional

Fuente: http://www.bomberosperu.gob.pelpo_muestra_esta.asp

\section{DISEÑO DEL ROBOT}

\subsection{Diseño mecánico}

Se utilizó un subsistema motriz tipo oruga con mayor capacidad de contacto con la superficie. Además, permite alcanzar un radio de giro nulo. Se consideró amortiguadores independientes en cada eje.

En la figura 2, se muestra la estructura mecánica del robot diseñado.

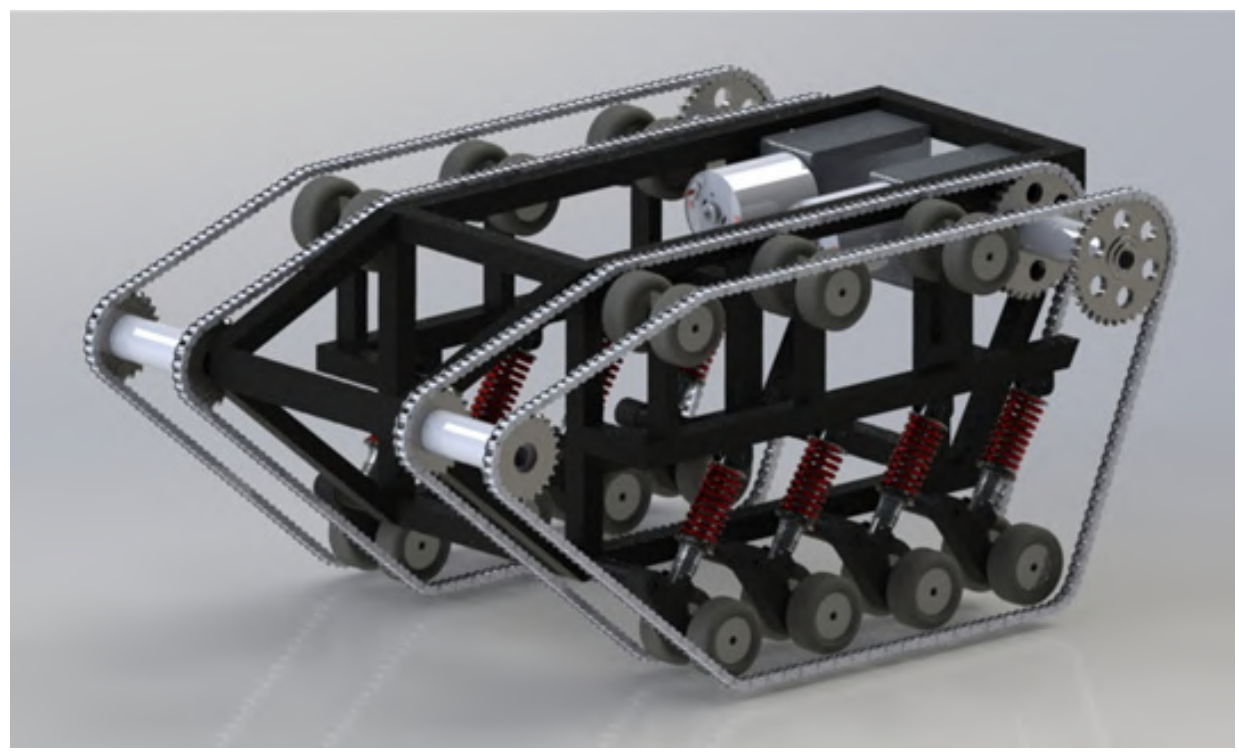

Fig. 2. Estructura mecánica del robot

Fuente: Elaboración propia 


\subsection{Diseño Eléctrico}

Se presenta en la tabla 1 los componentes del robot diseñado, sin considerar el emisor de señal y la cámara.

Tabla 1. Componentes del robot diseñado

\begin{tabular}{|l|c|c|c|}
\hline & Voltaje $(\mathrm{V})$ & Amperaje (A) & Cantidad \\
\hline Motor y controlador & 12 & 285 & 2 \\
\hline Módulo receptor & 6 & 0.4 & 1 \\
\hline Motor de paso y controlador & 12 & 6 & 2 \\
\hline
\end{tabular}

Fuente: Elaboración propia

Se determinó el consumo de corriente, lo que se muestra en la tabla 2.

TABla 2. Consumo de corriente total

\begin{tabular}{|l|c|c|}
\cline { 2 - 3 } \multicolumn{1}{c|}{} & Corriente de Arranque (A) & Corriente Nominal (Ah) \\
\hline Motor y controlador & 570 & 52 \\
\hline Modulo Receptor & 0.5 & 0.5 \\
\hline Motor de paso y controlador & 12 & 12 \\
\hline \multicolumn{1}{|c|}{ Total } & 582.5 & 64.5 \\
\hline
\end{tabular}

Fuente: Elaboración propia

En la figura 3, se muestra el esquema eléctrico del robot diseñado.

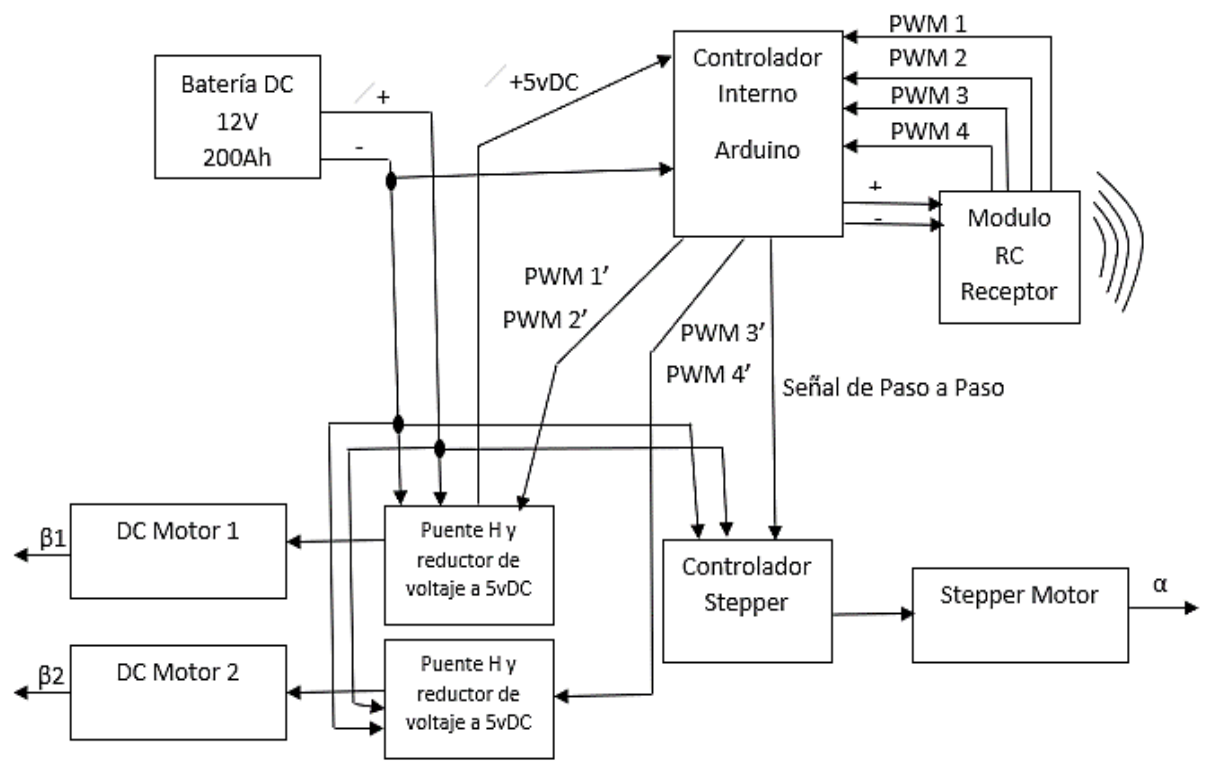

Figura 3. Esquema eléctrico del robot

Fuente: Elaboración propia 


\subsection{Diseño electrónico}

La figura 4 muestra la placa PCB diseñada con el software Proteus. que recibe la señal digital y controla el robot utilizando la plataforma Arduino.

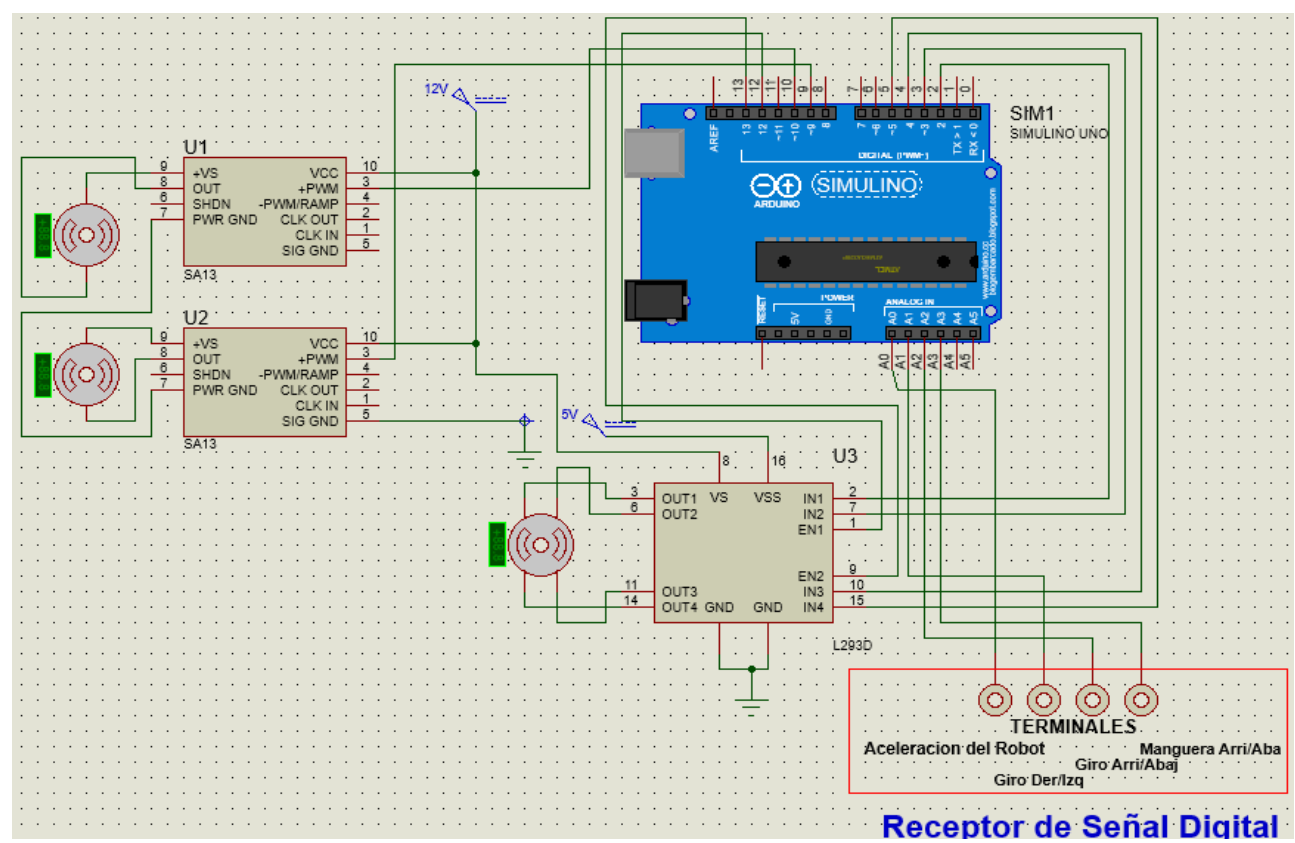

Fig. 4. Circuito electrónico del robot

Fuente: Elaboración propia

\subsection{Diseño informático}

Se utilizó tecnología de radio control y comunicación Wifi para la cámara térmica. Se usó un celular como receptor que presenta instalada una aplicación. En la figura 5, se muestra la cámara térmica.

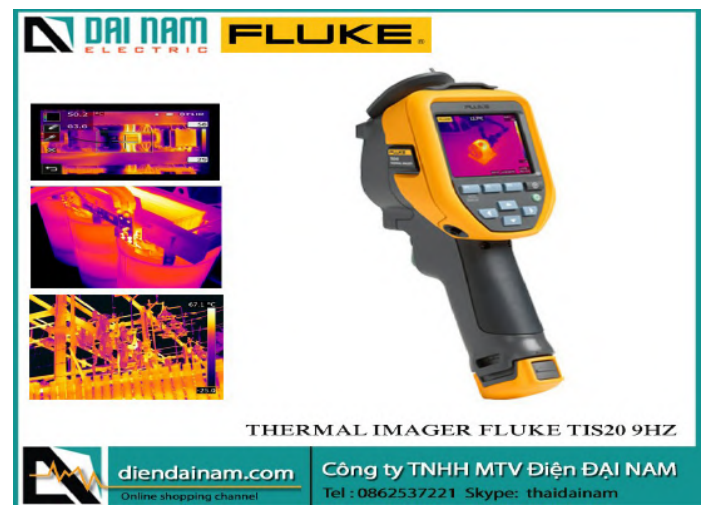

Fig. 5. Cámara térmica "Fluke Tis 20"

Fuente: https://diendainam.com/en/thermal-imaging-camera/121-thermal-imagers-fluke-tis20-9hz 


\section{IMPLEMENTACIÓN DEL ROBOT}

\subsection{Implementación mecánica}

Se implementó la estructura mecánica con tubos cuadrados LAC de $40 \mathrm{~mm}$ x $40 \mathrm{~mm}$. En la figura 5 , se muestra el sistema de suspensiones y las ruedas de soporte como tensores para la faja de oruga.

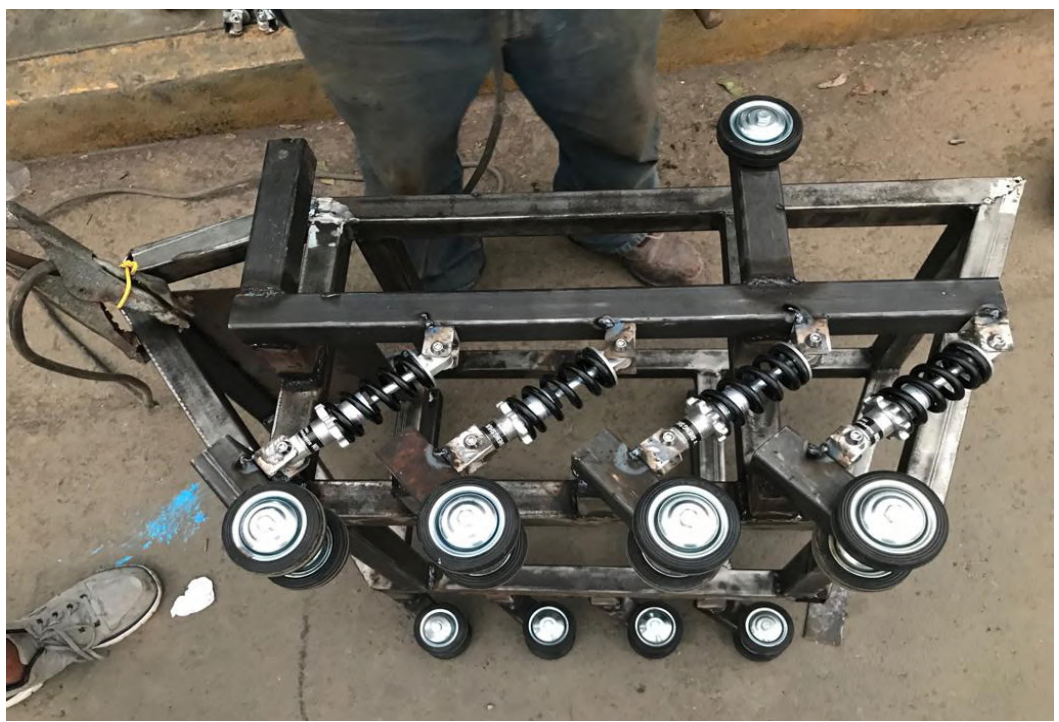

Fig. 6. Tensores para la faja de oruga

Fuente: Elaboración propia.

En la figura 7, se muestra la colocación de las fajas para la oruga del robot.

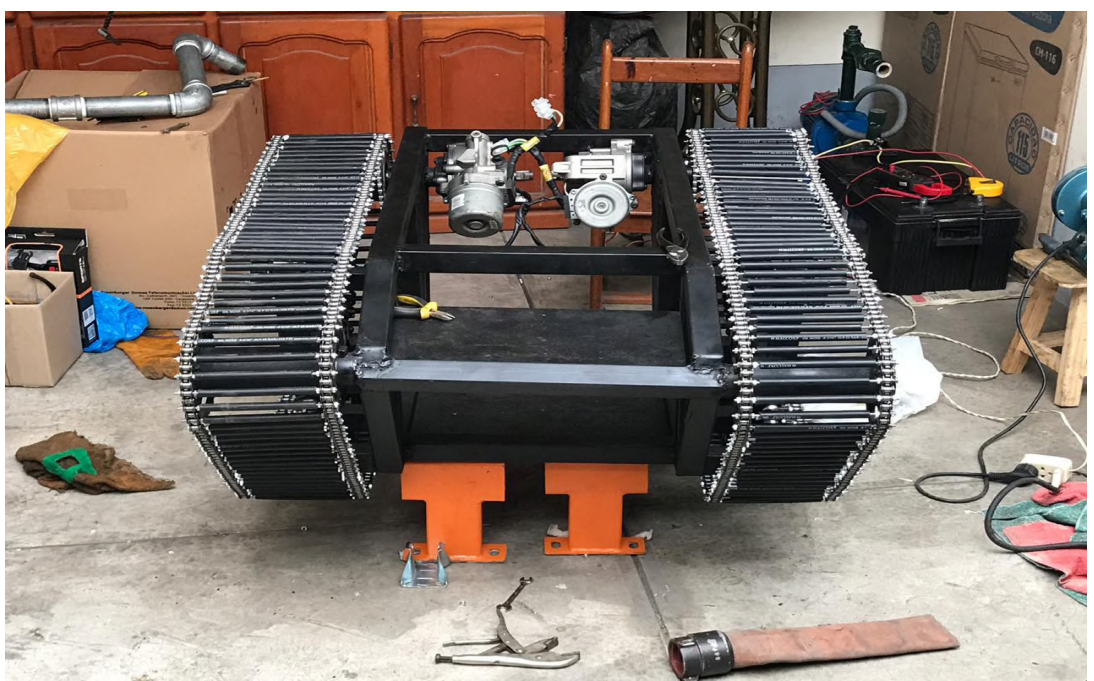

Fig. 7. Fajas de oruga del robot

Fuente: Elaboración propia 
Se instaló un acople trasero para el subsistema de disparo de agua del robot. En la figura 8, se muestra el sistema funcionalmente operativo en etapa de pruebas.

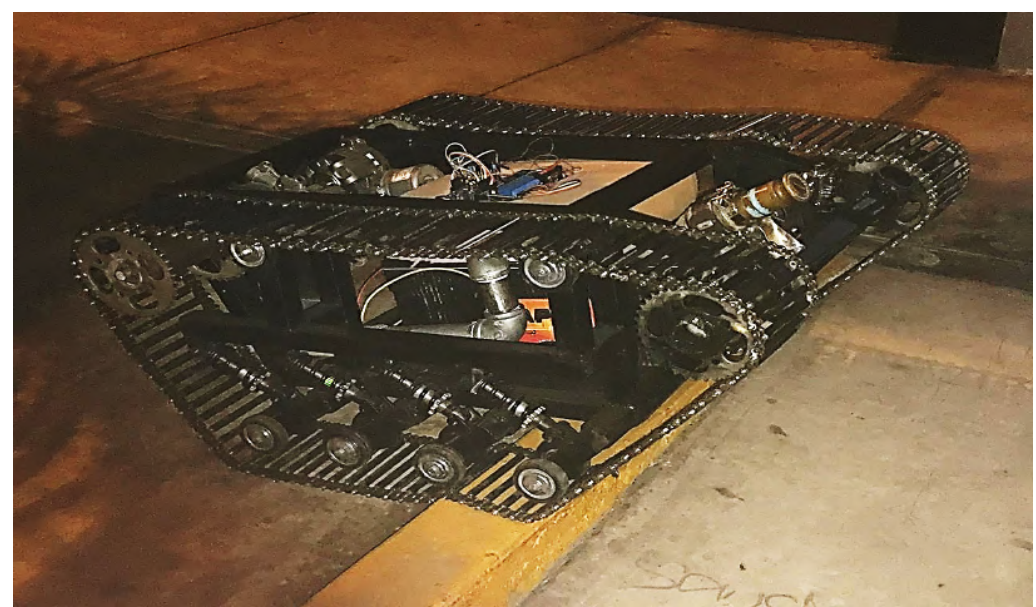

\section{Fig. 8. Robot concluido y operativo}

Fuente: Elaboración propia

\section{PRUEBAS Y RESULTADOS}

\section{Prueba 1}

Se probó las condiciones máximas de temperatura que soportaría el robot. Por ellos, fue necesario someterlo a un incendio provocado. Se demostró que el robot no presentó daños luego de ser expuesto a altas temperaturas, como se muestra en la figura 9.

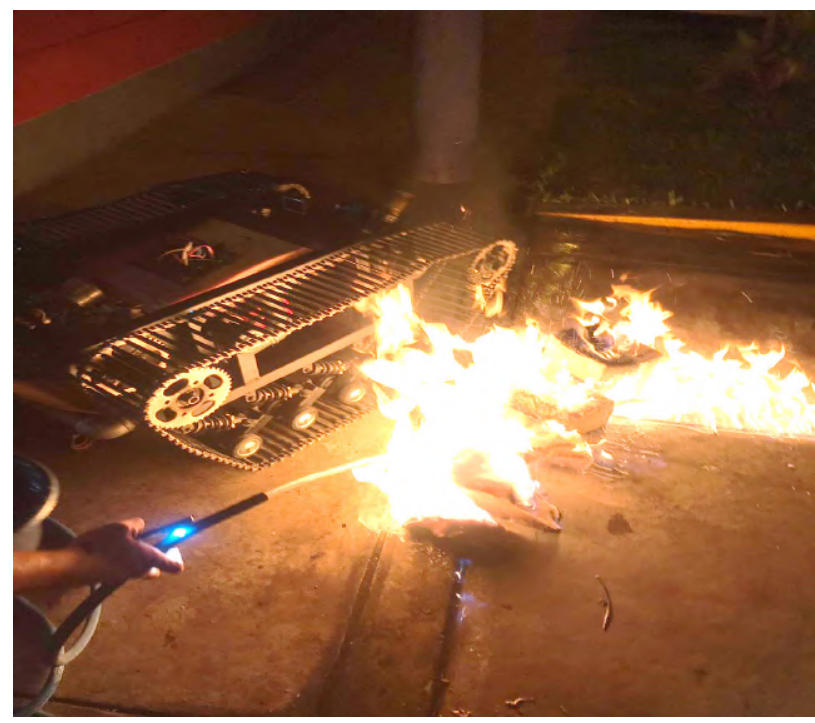

Fig. 9. Incendio provocado para prueba 1 Fuente: Elaboración propia 


\section{Prueba 2}

La prueba consistió el probar el subsistema de disparo de agua en un incendio provocado con una distancia menor a uno real. Durante la prueba, no se registró fugas de agua. Por su parte, el disparo no presentó vibraciones, fue preciso y resistió la presión requerida durante su realización, como se muestra en la figura 10 .

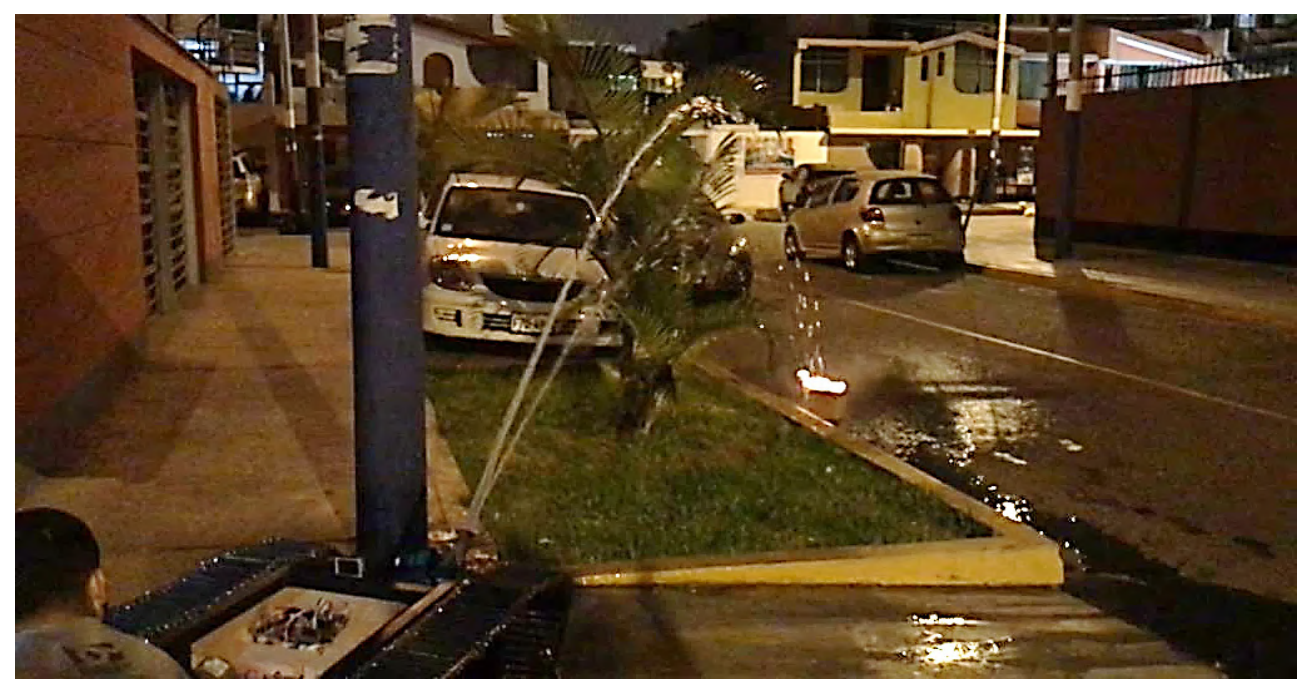

Fig. 10. Subsistema de disparo de agua en un incendio provocado

Fuente: Elaboración propia

\section{Prueba 3}

La prueba permitió evaluar altas y bajas temperaturas con la cámara térmica. En la figura 11, se muestra la imagen de térmica de una hornilla caliente.

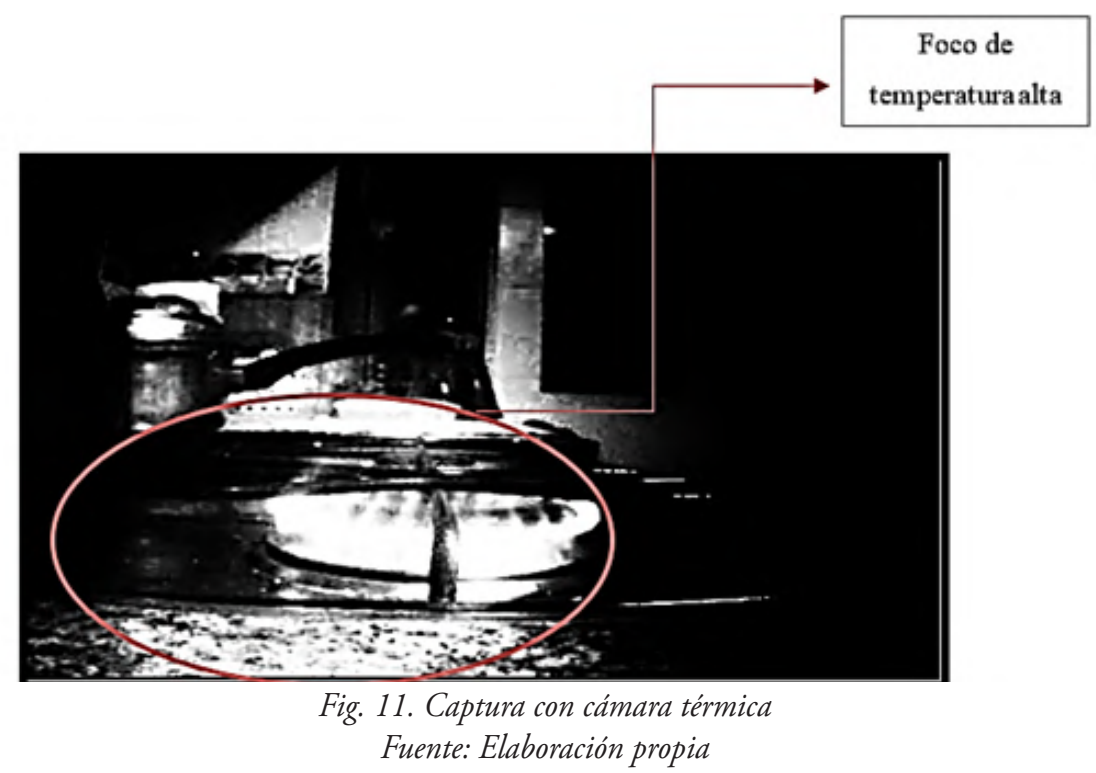




\section{Prueba 4}

La prueba final permitió probar el sistema de telecontrol. La figura 12, muestra la prueba de telecontrol del robot a cargo de un bombero voluntario.

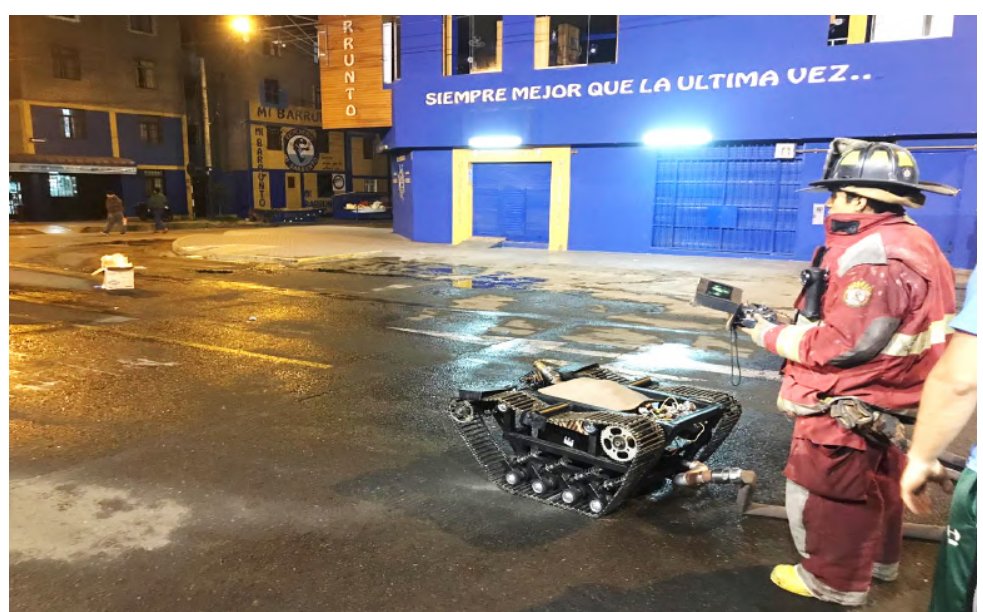

Fig. 12. Prueba de telecontrol del robot.

Fuente: Elaboración propia

\section{CONCLUSIONES}

Se puede afirmar que el robot telecontrolado fue estable durante su operación con disparos a altas presiones, dado que las pruebas 1 y 2 mostraron un buen rendimiento del sistema de amortiguamiento, y esto gracias a que no hubo fugas de agua ni vibraciones ni interferencias. Así mismo, a través de las pruebas 3 y 4, el robot demostró eficiencia en la expulsión de agua y calidad de imagen térmica, y distinguió puntos de altas temperaturas para la mitigación del incendio.

\section{REFERENCIAS}

[1] S. Argudo y A. Arpi. "Diseño y Construcción de un Robot Móvil Teleoperado para Asistencia en Operaciones de Alto Riesgo del Cuerpo De Bomberos”. Universidad Politécnica Salesiana, Ecuador. 2012.

[2] I. Aznarán y G. Reyes. "Aplicación de la Termografía Infrarroja en Tableros Eléctricos de Distribución para Mejorar la Seguridad y la Calidad de la Energía Eléctrica”. Universidad Nacional del Santa, Chimbote-Perú. 2016.

[3] A. Barrientos, L. Peñin, C. Balaguer y R. Araceli. Fundamentos de Robótica. (2a ed.). España, Madrid: McGraw-Hill. 2007.

[4] R. Borja y H. Bravo. "Diseńo e Implementación de un Robot Móvil Tipo Oruga para Exploración en Terrenos Irregulares". Control y Redes Industriales en la Escuela Superior Politécnica de Chimborazo, Riobamba-Ecuador. 2006.

[5] D. Drysdale. An Introduction to Fire Dynamics. $3^{\circ}$ Ed. U.K., John Wiley \& Sons, Ltd. 2001.

[6] A. Ollero. Robótica: Manipuladores y robots móviles. España, Barcelona: Marcombo. 2001.

[7] S. Pérez. "Prototipo de Robot Bombero Controlado a Distancia Mediante Dispositivo Móvil". Universidad de San Buenaventura Seccional Medellín, Medellín-Colombia. 2015. 